\title{
Osteoma de meato acústico externo: relato de nove casos e revisão de literatura
}

\section{Osteoma of external acoustic meato: report of nine cases and literature review}

\author{
José Ricardo G. Testa', Gilberto U. Pizarro², \\ Isabela M. De Vuono ${ }^{3}$, Maria Eugênia L. R. B. \\ Valeriano Neto $^{3}$, Fernando Barros ${ }^{4}$
}

\author{
Palavras-chave: osteoma, meato, acústico, \\ externo, hipoacusia. \\ Key words: osteoma, meato, acoustic, \\ external, hipoacusia.
}

\section{Resumo / Summary}

steo ma de meato acústico externo corresponde a um crescimento ósseo anormal e benigno, que se desenvolve abaixo da pele do conduto e pode causar obstrução total ou parcial do meato acústico externo. Formadeestudo: Estudo de série. Material emétodo: Ap resentamos neste estudo nove casos de osteoma de meato acústico externo. A idade dos pacientes variou de 23 anos (idade mínima) e 70 anos (idade máxima), sendo a média 40,6 anos. Desses nove pacientes, 5 eram do sexo feminino e 4 eram do sexo masculino. Resultado: Os sintomas mais comuns foram: hipoacusia, causada pela obstrução parcial ou total do conduto, otites externas recorrentes e também impactação de cerume de difícil remoção. Conclusão: A conduta nos casos apresentados foi cirúrgica, com boa evolução, não ocorrendo recidivas.

\begin{abstract}
T he osteoma of external acoustic meatus corresponds to an abnormal and benign bony growth, that is developed under the skin of the auditory canal and that can cause total or partial obstruction of the external acoustic meatus. Study design: Serie study. Material and method:We reported in this study nine cases of osteoma of external acoustic meatus. The patients' ages varied between 23 and 70 years old, being the average age 40,6 years. These patients were 5 of the feminine sex and 4 were of the masculine sex. Results: The more common symptoms were hipoacusia, caused by the partial or total obstruction of the canal, appealing external otitis and also the accumulation of cerumen deep in the external auditory canal with difficult removal. Conclusion: We recommended in the presented cases surgical treatment, which had good evolution, with no recidivism.
\end{abstract}

\footnotetext{
${ }^{1}$ Professor-doutor pela UNIFESP-EPM; Chefe do serviço de residência médica do Hospital Paulista de O torrinolaringologia.

2 Pós-graduando da Disciplina de O torrinopediatria da UNIFESP-EPM.

${ }^{3}$ Médica residente do Hospital Paulista de Otorrinolaringologia. ${ }^{4}$ Médico Otorrinolaringologista.

Instituição: Hospital Paulista de Otorrinolaringologia - Rua Dr. Diogo de Faria, 780 SP 04037-00 Tel/Fax: (0xx11) 5087-8700

Trabalho apresentado no $36^{\circ}$ Congresso Brasileiro de O torrinolaringologia, realizado de 18 a 23 de novembro de 2002 em Florianópolis, SC. Artigo recebido em 12 de dezembro de 2002. Artigo aceito em 13 de março de 2003.
} 


\section{INTRODUÇÃO}

Os autores apresentam nove casos de osteoma de meato acústico externo que são consideradas neoplasias benignas de crescimento lento.

Foram realizados exame clínico otorrinolaringológico, estudo radiológico com tomografia computadorizada que evidenciaram imagens densas e únicas obliterando parcial ou totalmente o meato acústico externo. Realizou-se também exames audiométricos que revelaram perdas auditivas condutivas.

Todos os pacientes foram submetidos a tratamento cirúrgico para remoção do osteoma. Dos nove pacientes operados, três apresentaram acúmulo epitelial do tipo colesteatoma de meato acústico externo obliterante, medialmente ao osteoma. Em todos os pacientes procedeuse o descolamento do epitélio sobre o osteoma para recobrir a área de inserção no meato acústico externo. Em um caso houve estenose do conduto que necessitou de um segundo procedimento com enxerto de pele.

Em todos os casos realizou-se estudo anatomo patológico que confirmou o diagnóstico. Até o momento não houve recidivas dos osteomas.

\section{Revisão de literatura e diagnóstico diferencial}

O steoma de meato acústico externo (MAE) corresponde a um crescimento ósseo anormal e benigno, que se desenvolve como uma massa semiesférica abaixo da pele do conduto e pode causar obstrução. Essa massa é dura e indolor. O s osteomas são sésseis e solitários com uma base estreita que conecta o osteoma ao osso do MAE. Geralmente são fixados nas suturas tímpano-mastóidea ou tímpano-escamosa. São tumores benignos, porém apresentam crescimento progressivo causando obstrução do MAE. Ao causar obstrução, irá promover uma perda auditiva condutiva e também otite externa recorrente.

O osteoma de meato acústico externo é uma lesão pouco freqüente, podendo ocorrer em qualquer idade sendo três vezes mais comum no sexo masculino ${ }^{1}$.

0 diagnóstico é feito através da história clínica do paciente, exame clínico otorrinolaringológico e exames de imagem como a tomografia computadorizada de ossos temporais. A tomografia computadorizada tem como objetivo enumerar e localizar as calcificações das paredes, avaliar o comprometimento do calibre do MAE, estudar a extensão em profundidade do osteoma e investigar a presença de patologias associadas de meato acústico externo e de ouvido médio².

Como diagnóstico diferencial de lesão óssea de meato acústico externo temos a exostose. Morrison, em 1948, realizou a primeira diferenciação clínica entre osteoma e exostose. ${ }^{3}$ Clinicamente, a exostose se caracteriza por uma elevação óssea com base larga, geralmente múltiplos e bilaterais acometendo o osso timpânico ${ }^{1}$. As exostoses são os tumores mais encontrados no MAE. Raros na infância, mais freqüentes no sexo masculino, apresentam como fator etiológico a exposição prolongada e repetida por água fria e salgada no MAE

Um estadiamento clínico para o osteoma foi proposto por Graham em 1982 - Estágio 1: tumoração visível ao examinador, porém assintomático para o paciente; Estágio 2: sintomático, porém controlável com tratamento conservador; Estágio 3: sintomático, necessitando de tratamento cirúrgico. ${ }^{4}$

Histologicamente, o osteoma de meato acústico externo é coberto por epitélio escamoso com o periósteo subjacente. Em sua estrutura interna apresenta discretos canais fibrovasculares, circundados por lamelas ósseas, com poucos osteócitos. ${ }^{5}$

As exostoses de meato acústico externo ficam sobre o periósteo sendo recobertas por epitélio escamoso. Ela é caracterizada pela presença de lâminas concêntricas de ossos subtemporal, com inúmeros osteócitos e poucos canais fibrovasculares. ${ }^{6}$

O tratamento dos osteomas pode ser conservador ou cirúrgico. 0 tratamento conservador consiste em prevenir otites externas e também perda condutiva, causados pelo acúmulo de epitélio escamoso descamado. Pode ser feito através da remoção do epitélio descamado e também com uso de antibióticos tópicos. 0 tratamento cirúrgico é realizado em pacientes com perda auditiva condutiva causada pela obstrução óssea e também em otites externas de difícil controle clínico. ${ }^{4} \mathrm{G}$ eralmente o osteo ma pode ser removido por via transmeatal com anestesia local. ${ }^{5} \mathrm{~A}$ abordagem retroauricular está indicada quando o osteoma está localizado medialmente ao istmo e quando é maior que 0 istmo. ${ }^{7} \mathrm{~A}$ remoção cirúrgica tem como objetivo restaurar o tamanho normal do meato acústico externo utilizando brocas e curetas na via de abordagem transmeatal com anestesia local. É muito importante preservar a pele do meato acústico externo para ser colocada no mesmo lugar permitindo cicatrizar e evitar a estenose do conduto. A cirurgia pode ser extremamente arriscada, com risco de lesão da membrana timpânica, nervo facial, orelha interna e bulbo da jugular necessitando que 0 cirurgião tenha experiência e treino suficientes para esta condição. ${ }^{8}$

A recidiva do osteoma, após sua completa remoção cirúrgica, ainda não foi relatada.

\section{APRESENTAÇÃO DE CASOSCLÍNICOS}

\section{Caso 1}

D.C., feminino, 31 anos, refere há 6 anos sensação de obstrução no meato acústico externo, com hipoacusia, otorréia intermitente e prurido auricular à esquerda. Refere antecedentes de otites supuradas aos 4 anos de idade.

Ao exame físico de orelha esquerda observou-se uma neoformação, dura ao toque com estilete, ocupando todo 0 
MAE, pele hiperemiada com descamação epitelial e otorréia (Figura 1).

Seu audiograma demonstrou uma perda auditiva condutiva à esquerda de aproximadamente $20 \mathrm{~dB}$.

A tomografia computadorizada de mastóide apresentou hipopneumatização e esclerose de células da mastóide esquerda, MAE esquerdo com contornos mal definidos e com áreas densas em seu interior.

Realizou-se tratamento cirúrgico sob anestesia geral, via retroauricular. Foi feito o descolamento da pele que cobria o osteoma e realizou-se o broqueamento do conduto para sua extirpação. Por via transmeatal, foi feita a aspiração da secreção e lamelas da descamção epitelial. A membrana timpânica estava retraída, espessada e hiperemiada. Por último, foi feito curativo com pomada e gelfoam.

o exame anatomopatológico revelou osso denso e revestido por epitélio escamoso.

Revisões periódicas revelaram estenose de meato acústico externo que necessitou de nova intervenção para enxerto de pele e restituir a permeabilidade. Controles subseqüentes revelaram-se sem anormalidades. A audiometria do pós-operatório estava normal.

\section{Caso 2}

R.S.P., feminino, 27 anos, refere há 8 anos hipoacusia à direita associada a otorréias e otalgias de repetição.

À otoscopia direita, observou-se neoformação endurecida obliterando todo o MAE. 0 exame físico à esquerda estava normal.

0 audiograma apresentou perda condutiva à direita de aproximadamente $40 \mathrm{~dB}$ e ouvido esquerdo normal.

Ao exame tomográfico, observou-se a presença de uma lesão óssea implantada no tecido mole do MAE à direita. Através do corte coronal, foi possível observar o pedículo do osteoma originando-se da parte óssea do conduto (Figura 2).

Foi realizado tratamento cirúrgico sob anestesia geral via retroauricular, realizando descolamento da pele do osteoma e broqueamento do tumor ósseo. Pelo MAE foi aspirado massa de descamação epitelial. A membrana timpânica encontrava-se espessada e retraída. Nos controles pós-operatórios não foram observadas alterações. 0 audiograma de controle demonstrou um pequeno gap residual de $10 \mathrm{~dB}$.

Ao exame anatomopatológico observou-se osso trabeculado denso com fibrose medular parcialmente revestido por epitélio escamoso.

\section{Caso 3}

I.V., masculino, 62 anos, refere hipoacusia há 15 anos, com dificuldades para remoção do cerume. Nega otorréias.

À otoscopia esquerda, observou-se MAE obliterado por tumor duro, fixado na sua porção anterior.

0 audiograma apresentou em orelha esquerda perda

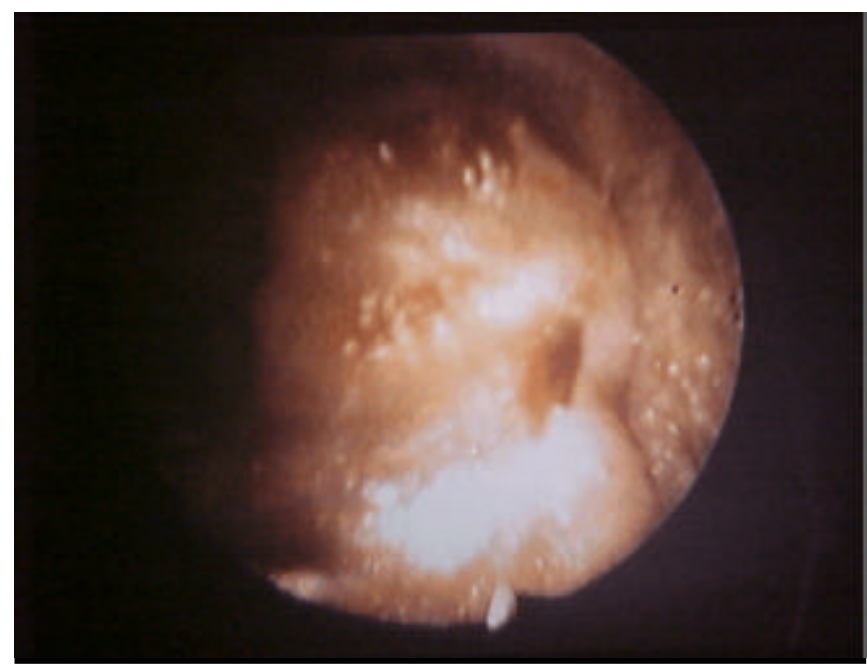

Figura 1. Neoformação óssea ocupando MAE.

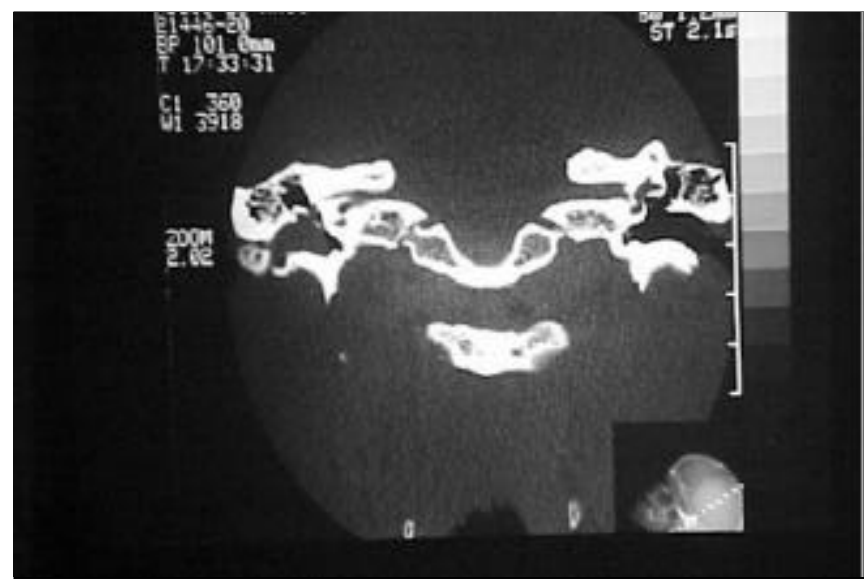

Figura 2. Osteoma originando-se da parte óssea do MAE.

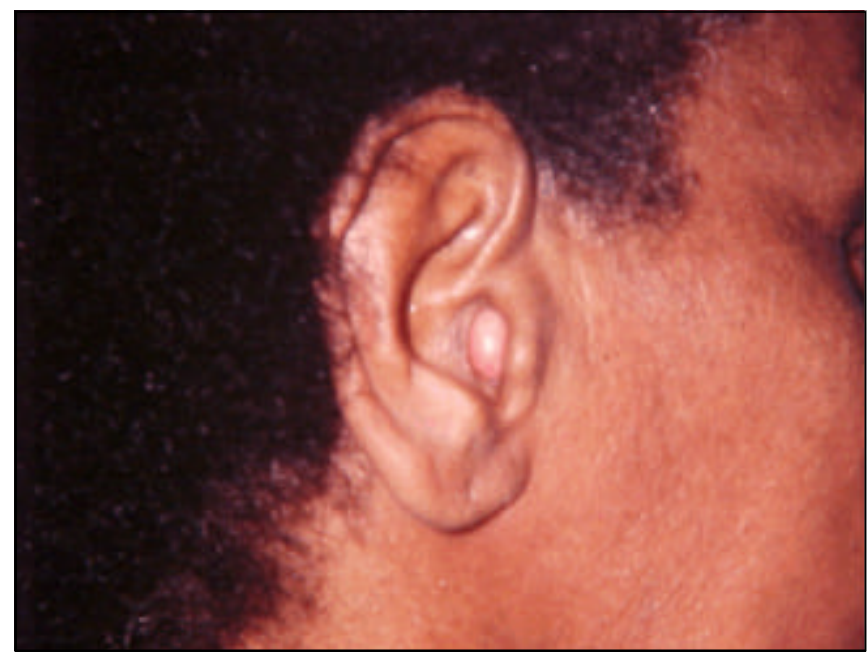

Figura 3. Osteoma de orelha direita ocupando todo o MAE. 
auditiva mista com disacusia neurossenssorial em agudos após $2 \mathrm{KHz}$ e gap de $25 \mathrm{~dB}$. Em orelha direita, ap resentou disacusia neurossenssorial em agudos após $2 \mathrm{KHz}$.

A tomografia computadorizada mostrou uma massa óssea única ocluindo o MAE esquerdo.

Realizou-se tratamento cirúrgico sob anestesia local mais sedação, via transmeatal. Feita a extirpação com pequena incisão na pele e fratura com cureta de sua base e broqueamento do resíduo. Feito curativo de MAE com pomada e gelfoam. Revisões pós-operatórias dentro da normalidade. Audiograma de controle com fechamento do gap.

O exame anatomopatológico confirmou o diagnóstico de osteoma, tendo sido observado neoplasia óssea benigna com trabéculas de osso lamelar maduro, revestido por epitélio escamoso sem atipias.

\section{Caso 4}

J.P.S., masculino, 28 anos, com queixa de hipoacusia bilateral há 10 anos, referindo também acúmulo de cerume com dificuldade para remoção. Esporadicamente apresentava otorréia e prurido auricular.

À otoscopia observou-se tumor endurecido e revestido por pele normal em ambos os condutos auditivos externos.

O audiograma apresentava uma perda condutiva bilateral, em orelha esquerda ao redor de $30 \mathrm{~dB}$ e em orelha direita ao redor de $20 \mathrm{~dB}$.

Ao exame de tomografia computadorizada, observou-se formações ósseas originadas da parte óssea do MAE bilateralmente, ocluindo a luz do conduto.

O paciente foi submetido a tratamento cirúrgico, sob anestesia geral, sendo feita a extirpação do osteoma com descolamento parcial da pele do MAE esquerdo e fresamento da base. Foi feito também a aspiração de acúmulo de descamação epitelial. Após 2 meses, realizou-se a remoção do osteoma do MAE direito da mesma forma.

No controle pós-operatório de ambos os lados durante 1 ano, não se observou recidivas. Houve fechamento do gap bilateralmente.

Ao exame anatomopatológico observou-se osso trabecular denso revestido por epitélio escamoso.

\section{Caso 5}

O.S., feminino, 60 anos, apresentava queixa de hipoacusia à direita há 15 anos, apresentando otorréias de repetição na infância.

À otoscopia de orelha direita, observou-se tumor duro ocupando o meato acústico externo direito fixado a sua parede anterior e superior (Figura 3).

Apresentava um perda auditiva a direita de aproximadamente $20 \mathrm{~dB}$ ao audiograma, enquanto a orelha esquerda estava normal.

A tomografia confirmava a presença do tumor de MAE direito fixo à parte óssea do MAE.
Realizou-se o tratamento cirúrgico sob anestesia local mais sedação, sendo feita a remoção do osteoma com descolamento parcial da pele do MAE direito por via transmeatal.

As revisões periódicas no pós-operatório durante 2 anos não apresentaram recidiva. 0 audiograma de controle apresentou fechamento do gap. patológico.

0 diagnóstico foi confirmado pelo exame anatomo-

\section{Caso 6}

G.G., masculino, 32 anos, apresentando hipoacusia à direita há 5 anos. Referia também prurido auricular, acúmulo de cerume de difícil remoção e otites externas de repetição.

À otoscopia apresentava obliteração do MAE direito por espessamento anular irregular subepitelial com membrana timpânica normal.

A audiometria não mostrava alterações.

A TC mostrava uma neoformação óssea comprometendo a luz do MAE direito (Figura 4).

0 tratamento de escolha foi a remoção da neoformação sob anestesia local, via transmeatal, com descolamento da pele do MAE e fresamento com remoção do espessamento ósseo com cuidado para não lesar a membrana timpânica.

O exame anatomopatológico confirmou o diagnóstico de osteoma de MAE direito.

No acompanhamento pós-operatório não houve recidiva.

\section{Caso 7}

E.V., masculino, 33 anos, com queixa de hipoacusia à esquerda há 10 anos.

À otoscopia apresentava tumor endurecido, ocupando parcialmente o MAE esquerdo.

À audiometria, observou-se perda auditiva condutiva leve à esquerda.

Realizou-se também TC, confirmando ser uma tumoração óssea originando-se do MAE.

O tratamento proposto foi a extirpação do tumor sob anestesia local via transmeatal.

O diagnóstico de osteoma foi confirmado pelo anatomopatológico. Não houve recidivas.

$\mathrm{Na}$ audiometria de controle no pós operatório, houve fechamento do gap.

\section{Caso 8}

S.V.S., feminino, 23 anos, com queixa de hipoacusia à direita, com impactação de cerume de difícil remoção. Evoluiu com piora do quadro, apresentando otites externas de repetição.

Apresentava tumoração endurecida obstruindo parcialmente o MAE direito.

$O$ audiograma demonstrava uma perda condutiva na orelha direita de $40 \mathrm{~dB}$. 


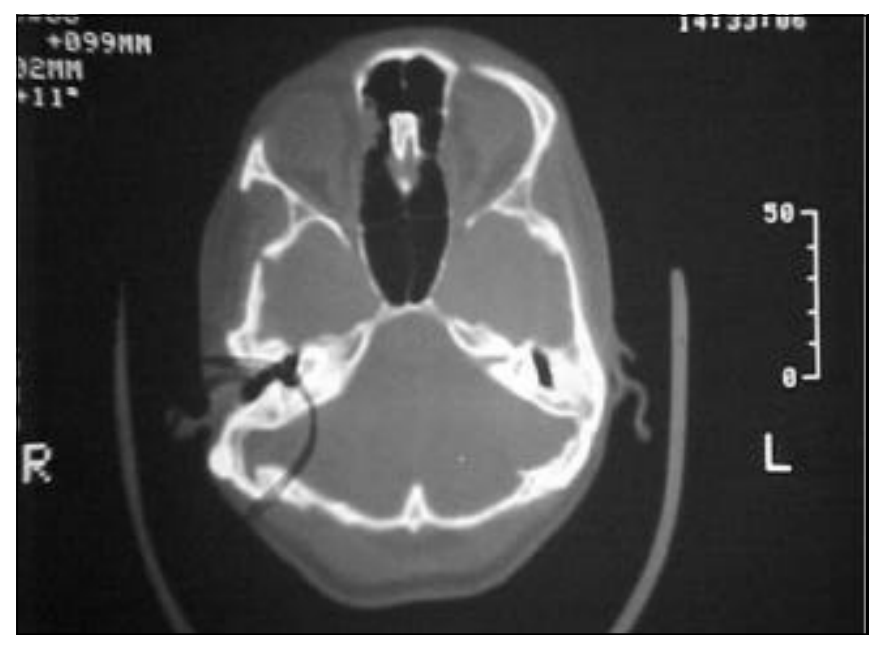

Figura 4. Neoformação óssea comprometendo a luz do MAE

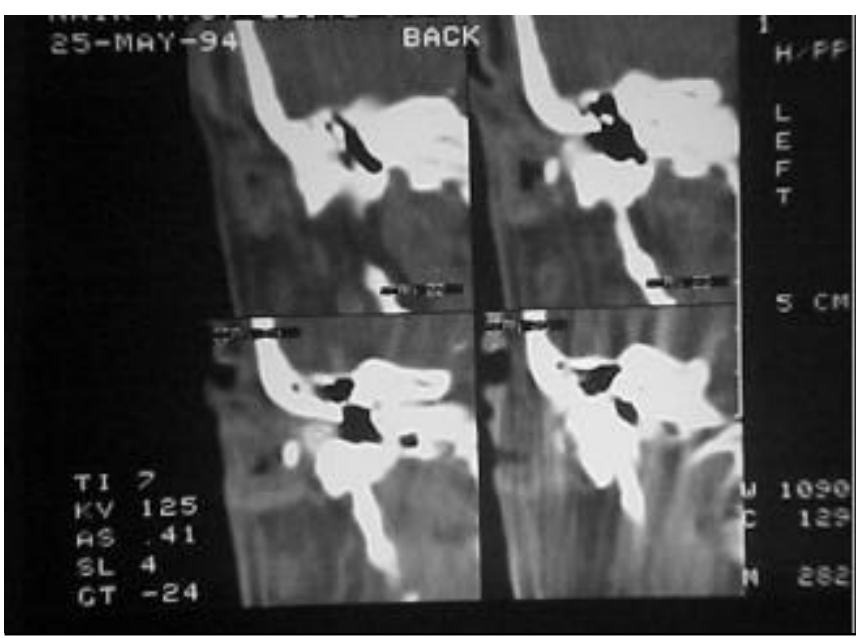

Figura 6. Ostemoa obstruindo parcialmente a luz do MAE direito.

Uma tumoração de constituição óssea foi observada na TC, obliterando parcialmente o MAE direito (Figura 5).

Realizou-se o tratamento cirúrgico, sob anestesia geral, por via retroauricular, observando-se um osteoma séssil na porção infero posterior do MAE direito, confirmando o diagnóstico de osteoma pelo estudo anatomopatológico.

No controle pós-operatório de 10 meses, houve fechamento do gap, não ocorrendo recidiva.

\section{Caso 9}

N.A.S.L., feminino, 70 anos, apresentava queixa de surdez bilateral, sendo pior em orelha esquerda (anacusia). Faz uso de prótese auditiva à direita há 10 anos. Refere

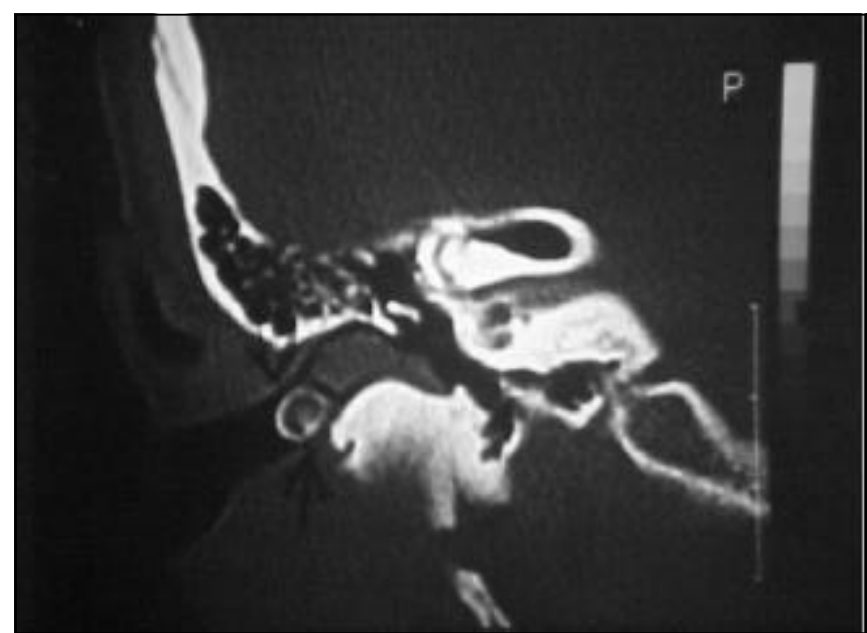

Figura 5. Osteoma obliterando a luz do MAE.

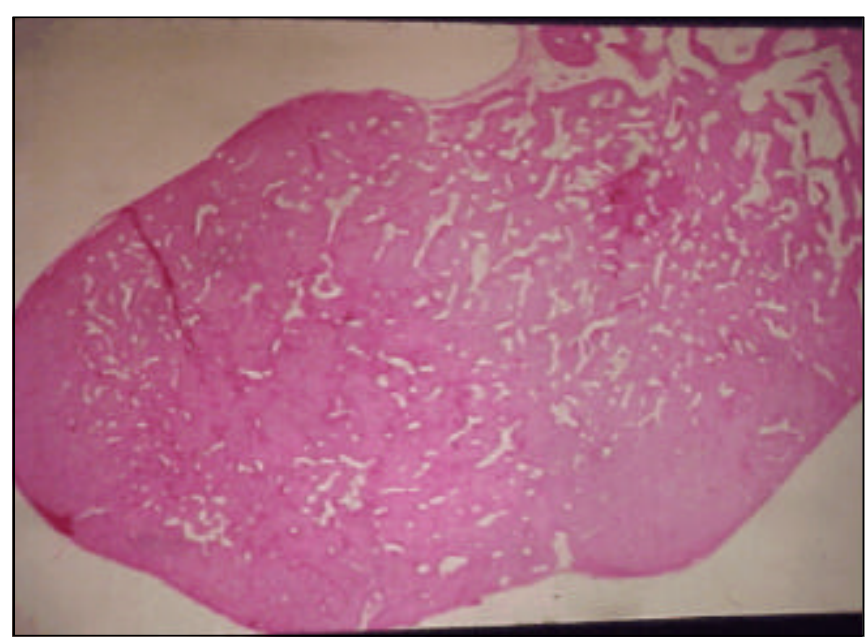

Figura 7. Anatomopatológico de osteoma: em sua estrutura interna, presença de discretos canais fibrovasculares, circundados por lamelas ósseas.

aumento dos episódios de otites externas e dificuldade para usar a prótese (não era possível adaptar o molde).

À otoscopia, tumoração endurecida de MAE direito obstruindo quase completamente a sua luz.

0 audiograma demonstrava uma anacusia à esquerda e uma perda auditiva mista à direita com gap de $25 \mathrm{~dB}$. O bservou-se na TC um lesão óssea diminuindo acentuadamente a luz do MAE à direita (Figura 6).

Feita a remoção cirúrgica do tumor, sob anestesia local, via transmeatal, confirmando o diagnóstico de osteoma pelo anatomopatológico (Figura 7).

No pós-operatório houve fechamento do gap, não havendo recidiva. 


\section{DISCUSSÃO}

De acordo com a literatura, os osteomas de MAE podem ocorrer em qualquer idade sendo 3 vezes mais comum no sexo masculino. ${ }^{1}$

Miziara et al. (1995) afirmaram que estas tumorações são infreqüentes antes da puberdade. Ohhashi et al. (1984) referem ocorrência mais comum entre as mulheres, na proporção de 2:1. ${ }^{9} \mathrm{Em}$ nosso estudo, dos 9 pacientes estudados obteve-se a média de idade de aproximadamente 40,6 anos, sendo a idade mínima de 23 e a idade máxima de 70 anos. Desse 9 pacientes, 5 eram do sexo feminino e 4 eram do sexo masculino.

Q uanto à etiologia dos osteomas, acredita-se que eles ocorrem em resposta à diminuição da temperatura no MAE. ${ }^{10}$

Todos os casos relatados nesse estudo correspondiam a osteomas de meato acústico externo. O steomas localizados fora do MAE são raros. Eggston e Wolff relataram sobre osteomas localizados na mastóide. ${ }^{11}$ Denia et al. apresentaram 8 casos de osteoma, sendo a localização mais comum a mastóide. ${ }^{12}$ O steomas localizados no ouvido médio já foram relatados. ${ }^{13,14} \mathrm{Em}$ 1995, Miziara et al. relataram um caso de osteoma localizado no conduto auditivo interno (CAI).

Todos os pacientes foram submetidos a tratamento cirúrgico, não apresentando nos controles pós-operatórios recidiva do osteoma. Apenas em 1 caso houve estenose do $M A E$, sendo necessária nova intervenção cirúrgica para colocação de enxerto de pele. Após a remoção completa do osteoma, recorrências não foram relatadas. ${ }^{7,15}$

\section{COMENTÁRIOSFINAIS}

O osteoma de meato acústico externo é uma patologia pouco freqüente, benigna, porém com crescimento progressivo, podendo assim tornar-se sintomático. Os sintomas mais freqüentemente relatados são a hipoacusia e otites de repetição que podem ou não serem controladas apenas com tratamento clínico. Nos casos resistentes ao tratamento clínico, opta-se pela abordagem cirúrgica. 0 diagnóstico deve ser confirmado pelo exame anatomopatológico para descartar outros tumores desta região.

\section{REFERÊNCIASBIBUOGRÁFICAS}

1. Noordzij JP, Arriaga MA, Stone AB. Differentiating Bony Lesions of the External Auditory Canal. Ear Nose Throat J 1995;74(1):4951

2. Bokowy $C$, Cadot $M$, Lelièvre G. Patología adquirida del oído externo. Encyclopédie Médico-Chirurgicale, Otorrinolaringología. París: Editions Scientifiques et Médicales Elsevier; 2001. p.1.

3. Morrison WW. Disease of the Ear, Nose and Throat. New York: Appleton-Century Crofts; 1948.

4. Graham MD, Kemink JL. Osteomas and exostoses of the external auditory canal-medical and surgical management. J Otolaryngol 1982;11(2):101-6.

5. Graham MD. Osteomas and exostoses of the external auditory canal. A clinical, histopathological and scanning electron microscopic study. Ann Otol Rhinol Laryngol 1979;88:566-72.

6. Miziara ID, Ianase MM, Bento RF. Osteoma de Conduto Auditivo Interno Associado a Surdez Súbita Contralateral. Rev Bras Otorrinolaringol 1995;61(5):396-8.

7. Sheehy JL. Diffuse exostoses and osteomata of the external auditory canal: A report of 100 operations. Otolaryngol Head Neck Surg 1982;90 (3 pt 1):337-42

8. Pulec JL, Deguine C. Osteomas of the external auditory canal. Ear Nose Throat J 1993;72(2):112.

9. Ohhashi M, Terayama $\mathrm{Y}$, Mitsui $\mathrm{H}$. Osteoma of the temporal bone - a case report. Nippon Jibiinkoka Gakkai Kaiho 1984;87:590-5.

10. Michaels L, Coucek S. Growth and pathology of the osseous ear canal and its epithelium. J Pathol 1988;154:100 A.

11. Eggston A, Wolff D. Histopathology of teh Ear, Nose and Throat. Baltimore: Williams and Wilkins; 1947. p.471.

12. Denia A, Perez F, Canalis RR, Graham MD. Extracanalicular osteomas of the temporal bone. Arch Otolaryngol 1979,105:7069.

13. Thomas R. Familial osteoma of the middle ear. J Laryngol Otol 1964;78:805-7.

14. Glassock ME, McKennan KX, Levine SC. Osteoma of the middle ear: a case report. Otolaryngol Head Neck Surg 1987;97:64-5.

15. DiBartolomeo JR. Exostoses of the external auditory canal. Ann Otol Rhinol Laryngol 1979;88(6)[suppl]61:1-20. 